\title{
Thanks to our reviewers in 2013
}

We would like to thank the following reviewers for their support by taking part in the peer-review process of the journal Amino Acids The Forum for Amino Acid, Peptide

A. Abell

S. Adams

S. Aksu

G. Aldini

B. Aleksiev

C. Alford

L. Alhonen

N. Amato

S. M. Ametamey

M. Anderluh

L. C. Andersson

H. Assaad

P. S. Azevedo

J. B. Arterburn

T. Baczek

N. Baganz

V. E. Baracos

J. J. Barchi

S. Bartesaghi

D. Becker

D. Beghelli

P. Beisswenger

J. Beltowski

B. Bequette

D. B. Berkowitz

Ł. Berlicki

J. Bernard

M. Bertolotti

T. Beryozkina
G. Biamonti

T. Bie

G. Biggio

B. Birnir

C. Bloch

N. Blondeau

N. Blot

P. Bonner

D. Boturyn

M. Brandsch

K. Brix

P. Brzezinski

D. Buisson

R. Burchmore

T. Burger

D. Burrin

N. Calcutt

P. Campíns Falcó

J. Cao

E. Capriotti

C. Carlini

E. Carneiro

R. A. Casero Jr.

C. Cativiela

J. Cavanaugh

M. Cervelli

G. Cevasco

S. H. Cheong

Y.-J. Chi and Protein Research in 2013. Their contribution and advice is essential is maintaining the high quality of the journal.
Y. Chiba

M.-J. Choi

B. Cipolla

S. Cobb

A. Coker

S. Colombatto

J. M. Conlon

C. Conte

G. Corzo

P. Costelli

K. Crailsheim

B. Creton

A. Culf

D. Cullen

A. da Silva

Z. Dai

Z. Dai

H. Daniel

P. Darriet

A. Davidoff

I. B. Dawid

R. Dawson

K. De Grave

H. De Groot

E. de Leeuw

M. Desforges

S. G. De-Simone

E. Deu Sandoval

N. Deutz 


\begin{tabular}{|c|c|c|}
\hline P. Di Simplicio & K. Hitomi & X. Li \\
\hline U. Diederichsen & R. Hoffmann & L. Li \\
\hline L. Dillon & G. Hortin & J. Li \\
\hline B. Dolenský & A. Hossain & M. Li \\
\hline H. Dolznig & T. J. Hou & X. F. Liang \\
\hline I. Drenjancevic & J. $\mathrm{Hu}$ & S. F. Liao \\
\hline J.-F. Dufour & T. Huang & C.-C. Lin \\
\hline E. Dzambazova & Y. Huang & H.-J. Lin \\
\hline M. Ebrahimi & N. Inguimbert & W.-W. Lin \\
\hline D. Echevarria & Ishii & Z. Lin \\
\hline R. Efremov & $\mathrm{T}$ Ito & A. Lippert \\
\hline A. El Idrissi & T. Ito & S. Liu \\
\hline J. Escobar & & B. Liu \\
\hline C. Esposito & S. Jacob & Y. Liu \\
\hline & R. Jaladanki & G. Y. Liu \\
\hline F. Facchiano & A. Jeukendrup & A. Llindahl \\
\hline I. Fajardo & J.-C. Jonas & \\
\hline C. Farinha & S. Jones & J. Ma \\
\hline D. Feith & C. Jonsson & Q. Ma \\
\hline J.-Q. Feng & D. Josic & X. Ma \\
\hline S. Feng & S. Jugé & N. Maier \\
\hline A. Fernie & C. Jungas & J. Marcinkiewicz \\
\hline J. Fernstrom & & M. Marina \\
\hline L. Fesus & C. Kahana & P. L. Martelli \\
\hline P. F. Fitzpatrick & L. Käll & L. Martens \\
\hline L. Floeter-Winter & R. Karuturi & R. Martinez-Manez \\
\hline W. A. Fogel & T. Kavanagh & G. Martra \\
\hline J. Forbes & I. Kawamura & N. Maruyama \\
\hline F. Franconi & Y. Kawata & G. Marverti \\
\hline N. Froger & T. Keinänen & H. Masaki \\
\hline N. Fukagawa & R. Kellner & T. Masaki \\
\hline A. Fuso & K. Kharbanda & J. L. Mascareñas \\
\hline & A. Khomutov & J. M. Mates \\
\hline H.-J. Gabius & H. Kimura & P. Matos \\
\hline B. Gabrielsson & N. King & T. Matsuda \\
\hline H. Gao & J. Kirchhoff & J. Matthews \\
\hline M. Gheorghiade & P. Koehler & K. Matuschewski \\
\hline T. Goldberg & H. Koepsell & C. Matute \\
\hline P. Goodyer & N. Koizumi & K. H. Mayo \\
\hline S. Gräslund & H. Kolmar & K. McCully \\
\hline M. Griffin & E. Kontny & R. Meijers \\
\hline O. Grygorenko & Y. Konttinen & O. Melnyk \\
\hline B. Gualano & R. Koopman & S. Menichetti \\
\hline M. N. Gupta & G. Krupitza & A. Michael \\
\hline J. Gurdon & D. Kyriakidis & J. Milner-White \\
\hline H. Gutiérrez-de-Terán & R. Lakshman & G. Minervini \\
\hline K. Hamase & G. Lalmanach & B. Mittendorfer \\
\hline P. Harris & M. Lämmerhofer & T. Miyazaki \\
\hline M.-P. Hasne & B. Lang & V. Monnier \\
\hline A.-E. Hassanien & S. Laufer & V. Montanari \\
\hline C. $\mathrm{He}$ & A. Lebedev & M. Morris \\
\hline J. Higley & L. Lecanu & L. Moruz \\
\hline T. Higuchi & D. Leslie & V. Mossine \\
\hline
\end{tabular}


J.-P. Moulinoux

A.-K. Mueller

H. Mukai

B. W. Murray

C. Muscari

T. Mutoh

R. Nagai

M. Nagl

K. Nakai

T. Narumi

H. Nicastro

E. Nice

E. Nicholson

K. Nishimura

Y. Nitta

A. Novelli

C. Nowicki

A. Ogunkoya

M. Ohkido

T. Oikawa

S. Oja

T. Ono

S. Oredsson

R. Oriá

M. Osta

C. Panagiotidis

S. Pantano

E. Papaleo

R. Papi

A. Papini

M. H. Park

E. Park

I.-K. Park

Y. Park

M. B. Passani

D. Pastore

A. Pastore

V. Pattabiraman

T. Patterson

A. Pegg

S. Pegoraro

V. Pehder

R. Penafiel

O. Perez

L. Persson

O. Phanstiel

F. Piao

G. Piccialli

G. Picolo

C. K. S. Pillai

E. Pilon-Smits
P. Pilowsky

M. Pischetsrieder

P. Pollard

M. Pongsavee

E. N. Prabhakaran

I. Pusterla

Y. Qi

M. V. Rajam

B. Rakitsch

S. Ramarathinam

P. Rameshwa

E. Randall

P. Rao

M. Raposo

R. Ratan

E. Razin

L. Reitzer

D. Rentsch

R. Rezaei

T. Riahi

P. Richomme

M. Ritzefeld

H. Roder

R. Roenicke

F. Rohrbacher

E. Ron

R. Rossi

K. A. Roubelakis-Angelakis

M. Rowan

I. Rubio Aliaga

M. Ruchko

C. Rupasinghe

G. Sahota

E. Salido

E.-K. Samer

M. Sammalkorpi

I. Sanchez

C. Sanmartín

R. Sardella

S. Sartori

T. Sato

G. Schaefer

P. Schaffer

D. Scheuer

S. Schildknecht

H. Schlüter

A. Scholey

G. Scholz

D. Senchina

S. Sengupta

D. Serafini-Fracassini
J. Sereikaite

P. Shah

G. Shah

L. Shantz

W. Shen

B. Sikorska

T. Silva

F. Simao

Z. Simões

K. Singh

P. Smialowski

I. Smith

W. Snedden

M. Sol Brassesco

A.-M. M. Soliman

J. Song

L. Spencer-Valero

K. S. Sreevenugopal

J. Steinert

M. Stipanuk

G. Subra

L. Szabados

T. Taku

A. Tan-Wilson

P. Tavladoraki

C. Tekwe

D. Telci

F. Tessier

B. Thiede

T. J. Thomas

I. Thondorf

P. Thornalley

D. Thwaites

C. Tian

A. F. Tiburcio

E. Tiligada

H. Tomitori

A. Torrado Agrasar

M. Torrent

P. Torrigiani

G. Tosi

D. Tourwe

D. Tsikas

V. Tumiatti

T. Uemura

T. Ulmer

A. Undas

A. Urbach

J. Van Goudoever

E. Vandermarliere

L. Vardy 

C. Ventura
W. Weckwerth
N. Yarlett
J. Vepsaläinen
S. Wienkoop
H. Yi-lin
J. C. Verster
B. Windshügel
Y. Yin
R. Villalonga Santana
D. Winkler
T. Yoshimura
P. Vinardell
O. Witard
S. Yu
K. Vinod
F. Wu
E. Voslarova
J.-Y. Wu
H. M. Wallace
Y. Wu
I. Walsh
M. Walsh
P. Wydro
Z. Wang
A. Wyse
X.-H. Xiao
C.-J. Wang
J. Xu
J. Wang
D. $\mathrm{Xu}$
M.-H. Xu
N. Zachara
M. Zaja
N. Zaki
C. Zhang
P. Zheng
Z. Zhou
P. Zhou
X.-D. Wang
S. Xu
H. Zhu
P. Zou
X. Wang
Y. Yamakoshi
A. Zulli
X.-Q. Wang
M. Watford
X. J. Yang
K. Watson
K. Yao
B. Zümreoglu-Karan 